\title{
LÍMITES AL DERECHO DE ACCESO A LA INFORMACIÓN CLÍNICA EN LOS CASOS DE ESTADO NECESIDAD TERAPÉUTICA Y ANOTACIONES SUBJETIVAS. ESPECIAL REFERENCIA AL SISTEMA ESPAÑOL
}

\author{
César A. Correa Martínez ${ }^{1}$ \\ Universidad Carlos III de Madrid ${ }^{2}$
}

Fecha de entrega: 27.02.2014

Fecha de evaluación: 18.03.2014

Fecha de aprobación: 26.05.2014

\section{RESUMEN:}

El derecho a la información que ha cobrado fuerza a lo largo del siglo XX, siendo uno de los pilares de la atención médica y de la relación médico - paciente, tiene algunas particularidades que, aun con reconocimiento constitucional y legal, dista de ser un derecho absoluto. Un ejemplo se encuentra en la Ley española de Autonomía del Paciente que consagra unos límites al derecho de información del paciente, específicamente dos de mucha importancia: el estado de necesidad terapéutica y las anotaciones subjetivas. Sin embargo la ley dejó el asunto abierto y dispuesto para ser debatido.

\section{PALABRAS CLAVE:}

Relación médico paciente, estado de necesidad terapéutica, anotaciones subjetivas, derecho de información, deber de informar.

\section{ABSTRACT:}

The right to information has gained importance throughout the twentieth century, one of the pillars of health care and the physician - patient relationship, has some peculiarities, even if they have constitutional and legal recognition, far from being a absolute right. An example is in the Spanish Patient Autonomy Act limits the right when patient information enshrined, specifically two very important: the state of therapeutic need and subjective annotations. However, the law left open and willing to be debated issue.

\section{KEYWORDS:}

Doctor-patient relationship, state of therapeutic necessity, subjective annotations, right to information, duty to inform.

\footnotetext{
${ }^{1}$ Abogado, especialista en Derecho médico de la Universidad del Rosario, Master en Derecho Público, de la Universidad Carlos III de Madrid.

${ }^{2}$ El presente estudio es un trabajo de investigación realizado en el Máster en Derecho Público de la Universidad Carlos III de Madrid, como consecuencia de la realización de la asignatura "Genética y Derecho", donde se realiza un tratamiento profundo del Derecho médico y la incidencia de la biomedicina.
}

REVISTA VIA INVENIENDI ET IUDICANDI, VOL. 8, No. 2

UNIVERSIDAD SANTO TOMÁS, BOGOTÁ

ISSN 1909-0528 


\section{Introducción y cuestiones previas}

El estudio que se pretende abordar, conlleva necesariamente tratar, con carácter previo, la conservación de datos de salud (refiriéndome a datos de salud como una generalidad que comprende datos médicos, sanitarios, genéticos, etc., en un conjunto sucesorio de información sobre el estado de salud de las personas contenidos en la historia clínica) y el deber de información del interesado - paciente. Sin embargo, los mismos serán estudiados de forma breve y solo por motivos explicativos, no como tema de fondo.

La información es una condición necesaria en toda práctica humana y ello comprende las situaciones médicas, al menos como una preocupación de las relaciones jurídica en la actualidad. De la buena información que el médico tratante dé al paciente depende, en gran medida, la libertad de éste para escoger dentro de las posibilidades terapéuticas la que el paciente considere la mejor. En ello se basa la autonomía del paciente ${ }^{3}$.

El punto de vista indicado, lo comparte la profesora León Sanz, cuando dice: "la autonomía del paciente, medida por la posibilidad de decisión, depende de lo que sabe" (LEÓN SANZ, 2004: 127). Y lo que el paciente sabe, en principio, es que está enfermo. También sabe que existe un equipo profesional dispuesto a ayudarle para salir del estado de disminución física o psicológica en que se encuentra con motivo de una enfermedad. Podemos suponer también que sabe o llegará a saber que la decisión que toma, depende de lo que el médico o equipo médico le informe. En este sentido, la obligación de informar al paciente es también una obligación moral.

Sin embargo esto no siempre fue así. Antes del siglo XX la medicina se basada en una práctica paternalista en la que el médico tomaba la decisión sobre el estado de

\footnotetext{
${ }^{3}$ Una autonomía limitada por la información que recibe y, que por tanto, es criticada pues no es posible saber hasta qué punto una persona es libre de tomar una decisión cuando se encuentre padeciendo dolores que le pueden llevar, inclusive, a la muerte.
}

REVISTA VIA INVENIENDI ET IUDICANDI, VOL. 8, No. 2 
salud del enfermo. Esta capacidad se fundaba en la especialidad del conocimiento médico adquirido por el galeno. En esta época, el paciente era considerado un ignorante de su estado de salud y por esta razón no se le permitía decidir sobre su bienestar. La mayor cantidad de información sobre pacientes y enfermedades que existen desde el siglo XIX -y hacia atrás-, data de la necesidad de registro práctico o educativo de la profesión médica; es decir, se toman notas terapéuticas y diagnósticas con el fin de conocer la enfermedad, su evolución y dejar constancia de las posibilidades desarrolladas en materia de salud para cada determinada época ${ }^{4}$.

En la actualidad, sin embargo, nos encontramos frente al debate sobre el uso y propiedad de la Historia Clínica y en general de la información del paciente. La cuestión del acceso y uso de la información se ha transformado en algo más que el mismo deber de información, pues aunque éste siga siendo asunto importante, poco a poco está siendo superado al menos en lo fundamental.

Sobre la propiedad de la información médica o de salud el consenso gira en torno a que dicho derecho le corresponde al paciente con algunas características propias en cuanto al acceso y uso de esa información por él o por terceros interesados. Esto es así, dado que se considera que la información clínica cumple con una función principal, cual es, lograr el mayor beneficio en el tratamiento de la enfermedad y en consecuencia, lo que reporte más beneficio al paciente. Todas las demás funciones le son consideradas accesorias.

En efecto, en el ámbito español, se debe destacar que la ley 41/2002, de 14 de noviembre, básica reguladora de la autonomía del paciente y la información clínica

\footnotetext{
${ }^{4}$ Así, desde las primeras Historias Clínicas de los textos hipocráticos en las que se encuentran un total de 48 registros de enfermos y heridos de guerra con el tratamiento recibido, pasando por Galeno que clasifica con la información clínica recopilada las enfermedades en géneros y especies, posteriormente los Consilium, textos de médicos de la época que llegan a las universidades de final de la edad media en que el registro de enfermedades se hace necesario para complementar el estudio teórico de las incipientes facultades de medicina y la Observatio del renacimiento hasta las teorías más modernas en que el registro médico (por ejemplo con Sydenham) cumple una función educativa. Ver: FOMBELLA Posada, María José y CEREIJO Quinteiro, María José (2012); Historia de la Historia Clínica, en Galicia Clínica 2012; 73 (1): 21-26.
}

REVISTA VIA INVENIENDI ET IUDICANDI, VOL. 8, No. 2

UNIVERSIDAD SANTO TOMÁS, BOGOTÁ

ISSN 1909-0528 
(en adelante LAP) otorga a los centros de salud, principalmente, la guarda de la información en ficheros que cumplan con unos requisitos que permitan asegurar la confidencialidad de dicha información ${ }^{5}$ Así por ejemplo, el artículo 14.2 de la citada disposición indica que "cada centro archivará las historias clínicas de sus pacientes, cualquiera que sea el soporte papel, audiovisual, informático o de otro tipo en el que consten, de manera que queden garantizadas su seguridad, su correcta conservación y la recuperación de la información".

De todo ello queda demostrado que la confidencialidad es ese elemento que prevalece en la relación médico - paciente. Cosa distinta cabe decir del uso de la citada información. Además del mismo paciente, que en casi la totalidad de los casos tiene derecho de acceso y disposición, la citada norma legal permite que accedan a ella los profesionales de la salud que tengan relación directa con el diagnóstico y tratamiento, el personal administrativo y al personal que tenga que hacer vigilancia y control, entre otros ${ }^{6}$.

Sin embargo, hay que aclarar que el acceso a la información del paciente no significa el acceso a toda la información que consta en el mismo por parte del titular del expediente, pues existen determinados datos que, al menos, en las más de las veces y mientras sea posible, se deben anonimizar. Esta previsión se entiende contenida en el "Convenio relativo a los derechos humanos y la biomedicina" que dispone en su artículo 10.1 el derecho de los ciudadanos a que se respete su privacidad cuando de su información médica se trate.

Esta previsión fue adoptada por la legislación española en la ley 41/2000 que sitúa en el artículo 13.1 el derecho del paciente a acceder a la historia clínica y a obtener

\footnotetext{
${ }^{5}$ Por eso es un derecho de propiedad difuso.

${ }^{6}$ El artículo 16 de la LAP regula los usos de la HC. Dentro de ellos está el 16.1 sobre uso asistencial, 16.2 sobre el uso de los médicos que asistan al enfermo, 16.3 de acceso con fines judiciales, epidemiológicos, de salud pública, 16.4 sobre acceso del personal administrativo y otros de acuerdo a sus funciones, 16.5 sobre el uso del personal sanitario acreditado con funciones de vigilancia y control.

${ }^{7}$ Convenio de Oviedo (Convenio Europeo sobre los derechos humanos y la biomedicina) de 4 de abril de 1997 del Consejo de Europa.
}

REVISTA VIA INVENIENDI ET IUDICANDI, VOL. 8, No. 2

UNIVERSIDAD SANTO TOMÁS, BOGOTÁ

ISSN 1909-0528 
copia de ella. Al efecto el mismo dispone textualmente que: "Los usuarios y pacientes del Sistema Nacional de Salud, tanto en la atención primaria como en la especializada, tendrán derecho a la información previa correspondiente para elegir médico, e igualmente centro, con arreglo a los términos y condiciones que establezcan los servicios de salud competentes“.

Entonces admitimos, y sin mayor sorpresa, que aunque esa información sea del paciente no es él quien la tiene bajo su custodia $^{8}$. Esto se debe a que al ser la información de salud una recopilación de datos sensibles (según la Ley 15/1999, de 13 de diciembre, Orgánica de Protección de Datos de carácter personal (en adelante, LOPD) con finalidad compleja, necesita cuidados especiales para su conservación. Cuando me refiero a la finalidad compleja auguro la principal función de la guarda de la información, el estado de salud de las personas en los centros sanitarios: que se le dé continuidad a los tratamientos del paciente. Pero además, a estos datos pueden acceder otras entidades públicas, siempre que una ley específica así lo autorice, para, por ejemplo verificar el estado de salud pública de la población, epidemiología, investigaciones criminales, docencia, etc. Este es por ejemplo, la autorización que se contiene en la Ley 16/2003, de 28 de mayo, de cohesión y calidad del Sistema Nacional de Salud.

Por esta razón, la legislación es muy exigente en cuanto a los requisitos para la conservación de dicha información. Estas medidas de seguridad están contempladas, entre otras, en la Directiva 95/46/CE relativa a la protección de las personas físicas en lo que respecta al tratamiento de datos personales y a la libre circulación de estos datos, en la Recomendación $R$ (97) 5, de 13 de febrero, del comité de ministros del Consejo de Europa a los estados miembros, sobre protección de datos médicos y en la ya citada LOPD -artículo 9-. Todas ellas, exigen la adopción de medidas, cuidado y organización apropiadas que impidan el

\footnotetext{
${ }^{8}$ La información clínica debe ser recopilada de forma imparcial y guardarse en condiciones de calidad que permitan su correcto almacenamiento.
} 
acceso no autorizado a esa información a quien no tenga interés en ella; es decir que protegen la confidencialidad y la integridad de los datos de salud.

Esta introducción, sirve para abordar el tema de fondo del presente trabajo cuya pregunta se centra, como hemos destacado, en determinar en qué casos el paciente tiene vetado el acceso a la información médica que consta en su historia clínica. Dentro de los casos que puedan limitar el acceso, la legislación española -LAPrecoge dos supuestos: a) el estado de necesidad terapéutica y b) las anotaciones subjetivas.

\section{Necesidad terapeútica}

El derecho a la información personal tiene una estrecha relación con los derechos fundamentales de las personas, en especial con el Derecho a la vida, a la integridad $y$, en el caso colombiano, a la salud ${ }^{9}$. Se trata de un derecho de cada persona y por ello, en cuanto afecta sus intereses individuales, ésta puede solicitar ser informado o no (el elemento positivo o negativo de la decisión) del estado de salud. En este caso, nos encontramos con el Derecho a no saber, como un derecho perfectamente reconocido en la legislación española en el artículo 9.1 de la citada LAP. En efecto, en dicho precepto se indica que "la renuncia del paciente a recibir información está limitada por el interés de la salud del propio paciente, de terceros, de la colectividad y por las exigencias terapéuticas del caso. Cuando el paciente manifieste expresamente su deseo de no ser informado, se respetará su voluntad haciendo constar su renuncia documentalmente, sin perjuicio de la obtención de su consentimiento previo para la intervención".

Sin embargo, es preciso destacar, que el acceso o no la información clínica, como todo supuesto de acceso a la información, no es un derecho absoluto y dentro de

\footnotetext{
${ }^{9}$ Con la sentencia $\mathrm{T}-760$ de 2008 la Corte Constitucional colombiana elevó a rango fundamental el derecho a la salud como derecho autónomo y a partir de allí se funda una doctrina jurisprudenial colombiana actual en ese sentido.
}

REVISTA VIA INVENIENDI ET IUDICANDI, VOL. 8, No. 2

UNIVERSIDAD SANTO TOMÁS, BOGOTÁ

ISSN 1909-0528 
las excepciones a que el paciente conozca la información de su historial médico, hay dos principalmente. Una de ellas, como ya dije, es el estado de necesidad terapéutica.

Sobre el estado de necesidad terapéutica (en adelante, ENT), el profesor Murillo de la Cueva, indica que "(...) es el que se da cuando razones objetivas ponen de manifiesto que el conocimiento por el paciente de su situación puede perjudicar de manera grave su salud" (Ripoll Carulla et al., 2006: 37).

En este sentido, se puede recordar que el estado de necesidad terapéutica a lo largo de la historia del siglo XX -después de la crisis de la visión paternalista de la medicina-, fundamenta su existencia en el humanismo con que deben ser tratadas las personas que tienen enfermedades fatales y cuyo conocimiento de éstas pueda afectar su nivel de vida ${ }^{10}$ o el desarrollo propio de la enfermedad. Esta posición, sin embargo, no ha sido bien recibida pues, para unos, estamos ante uno de los rezagos del paternalismo imperante en la relación médico paciente desde principios de siglo $\mathrm{XX}$ en la que el médico tomaba una decisión a nombre del paciente. Así, por ejemplo, el autor Oliva Blásquez critica este paternalismo de la siguiente manera:

\begin{abstract}
“¿Puede el médico privar al paciente, por motivos humanitarios o de compasión, de su derecho a ser informado del diagnóstico de su enfermedad? Históricamente se concedía en estos casos al médico el denominado 'privilegio terapéutico', eufemismo de claro corte paternalista con el que se justificaba la mentira piadosa que el médico contaba al paciente con la finalidad de evitar que se enfrentara a un diagnóstico final" (OLIVA BLÁSQUEZ).
\end{abstract}

\footnotetext{
${ }^{10}$ Haré una breve referencia a las diferencias, que parecen haber sido adoptadas por la LAP entre el privilegio terapéutico y la necesidad terapéutica.
}

REVISTA VIA INVENIENDI ET IUDICANDI, VOL. 8, No. 2

UNIVERSIDAD SANTO TOMÁS, BOGOTÁ

ISSN 1909-0528 
Desde este punto de vista, la limitación al deber de información al paciente no puede formar parte de la discrecionalidad del médico, sino que por el contrario, es conveniente y necesario que se encuentre recogido en una disposición legal voluntad del legislador en tanto representante de la soberanía popular-. Dicha disposición, será en su caso, la que debe regular los distintos supuestos, siempre mínimos, en que se produzcan la necesidad terapéutica.

De acuerdo con el profesor Oliva Blásquez, la actual LAP omitió hacer referencia al "privilegio terapéutico" por este motivo y lo sustituyó por el término de "necesidad terapéutica", término a su juicio más representativo. En efecto, desde su punto de vista, las "evidentes connotaciones negativas que éste tiene ${ }^{11 " ~ h i c i e r o n ~ n e c e s a r i o ~}$ dotar la excepción de verdaderas razones objetivas para poder esgrimir su utilización.

De ahí que podamos encontrar una diferencia razonable entre la necesidad terapéutica y el privilegio terapéutico, pues el segundo caso, utilizado históricamente, se basa en razones valoradas por el médico de acuerdo a un criterio subjetivo, a su creencia personal de evitarle al paciente un mayor daño. Sin embargo en el segundo, las razones, siempre mínimas, vienen determinadas por el marco normativo establecido.

La necesidad terapéutica que la LAP menciona, que ha sido confundida muchas veces con el privilegio terapéutico o con la ortotanasia -en estos dos últimos casos son criterios utilizados en pacientes con enfermedades terminales-, ha tenido un tratamiento distinto con respecto a estas dos. En tal sentido, la sección tercera del Consejo de Estado Colombiano se refirió al privilegio terapéutico de la siguiente manera:

\footnotetext{
${ }^{11}$ Palabras del autor, página 7 párrafo último. 
"la doctrina nacional se inclina por considerar que se debe relevar al médico del deber de información en aquellos eventos en los cuales "por circunstancias de carácter psicológico, el conocer los riesgos o las implicaciones de un tratamiento o de una intervención quirúrgica afectaría sus posibilidades de éxito y de recuperación (privilegio terapéutico)"; o cuando el destinatario de la información es otro profesional de la especialidad respectiva; el paciente ha sido sometido al mismo tratamiento en repetidas ocasiones, sobre el cual fue informado y dio su consentimiento; cuando el paciente, por su propia iniciativa, de manera anticipada y reflexiva, renuncia de manera expresa a su derecho a ser informado, y cuando resulta inexorable e inaplazable una determinada intervención (urgencia médica)."12

Pero hemos visto las diferencias que se suscitan entre una y otra.Así también lo ha mencionado Cristina Gil Membrado para quien:

"como tal privilegio, únicamente puede ser utilizado en circunstancias excepcionales y corresponde al profesional sanitario cerciorarse del grado de perjuicio ocasionado y que en ningún caso éste sea menor al producido por el quebrantamiento de un pilar fundamental en el tratamiento de datos personales, como es el derecho de acceso del interesado a la información que a su persona haga referencia" (GIL MEMBRADO, 2010: 251).

El privilegio terapéutico entonces, siendo que limita un derecho fundamental del paciente, tiene su crisis cuando en la actualidad toda diferencia entre el "estado de salud" y los "derechos fundamentales del paciente", debe beneficiar a éste y en ese

\footnotetext{
${ }^{12}$ Sentencia 15737 de 2008. Sala Tercera, Consejo de Estado de Colombia. El Consejo de Estado en Colombia funciona como máximo órgano jurisdiccional de la justicia contencioso administrativa. 
sentido se orienta la doctrina médico-sanitaria actual. El deber de información, al ser uno de los cimientos de la relación médico paciente, prima sobre las valoraciones subjetiva del médico o equipo médico. Por eso, la respuesta dada en la LAP fue la de aclarar que tal situación de omisión de información debe darse cuando existen razones objetivas y en tal sentido esas razones objetivas siempre serán aquellas que, a día de hoy, determine la medicina basada en la experiencia.

En España, la LAP consagró la necesidad terapéutica en el artículo 5.4. de la siguiente manera: "...el derecho a la información sanitaria de los pacientes puede limitarse por la existencia acreditada de un estado de necesidad terapéutica", y a continuación indica qué se debe entender por tal: (...) la facultad del médico para actuar profesionalmente sin informar antes al paciente, cuando por razones objetivas el conocimiento de su propia situación pueda perjudicar su salud de manera grave.

El cambio en la LAP, resulta muy relevante, dado que estipula las razones objetivas como criterio identificador de los "estados de necesidad terapéutica" y de esta forma se limita y se le determinan unas reglas claras al equipo médico del supuesto en los que puede negar información al paciente. Se aprecia de esta forma que se produce una ruptura de los principios que orientan la relación médico - paciente, pero que repitiendo lo que dice la LAP, se hace en búsqueda del mayor beneficio de la persona y también de los profesionales de la salud, que ven de esta manera, concretados los supuestos en los cuales pueden omitir ofrecer información al paciente, sin que se quebrante otras normas o principios recogidos, como el derecho a acceder a la información clínica por parte del paciente.

El primer aspecto que encontramos es que estamos ante una posibilidad contenida en la ley. Hemos visto entonces que la decisión de suspender los derechos del paciente a ser informado se encuentran, de forma razonablemente fundamentada, en la ciencia médica (en la medicina basada en la experiencia), aunque no sabemos

REVISTA VIA INVENIENDI ET IUDICANDI, VOL. 8, No. 2 
qué tan exclusiva es dicha posibilidad, pues siempre se discutirá hasta qué punto es posible esta facultad del médico tratante. Sin embargo es lógico que todo aspecto de la ciencia que afecte los valores individuales mayores (vida, justicia y libertad) defendidos por el humanismo, será objeto de un fuerte debate.

Aunque no existe claridad sobre este límite a la información, lo que genera miedo en el personal sanitario al momento de limitar la información al paciente por un "estado de necesidad terapéutica"13 ${ }^{3}$, es la restricción al deber de informar del médico y al derecho del paciente de ser informado sobre su estado de salud cuando se sospeche estar ante una enfermedad grave que le perjudique su salud y ello siempre será un asunto confuso y gris. En este sentido, una solución a este problema lo puede constituir la existencia en los centros asistenciales en salud de Comités de Ética Asistencial (en adelante, CEA), Comités que pueden ofrecer al personal sanitario una ayuda y orientación sobre cuándo en el caso concreto existe un estado de necesidad terapéutica. Se trataría en este caso, de un criterio a seguir, o de un criterio auxiliar a la toma de decisión del equipo médico o personal sanitario $^{14}$.

En efecto, en este sentido, cabe recordar que los CEA tienen asignados y reconocidas el desarrollo de tres funciones principales: formación ética, análisis de casos y formulación de directrices. De las tres funciones indicadas, nos interesa la segunda de ellas, es decir, el análisis de casos. Sería casualmente, en estos supuestos, en los que el equipo médico, el profesional o el personal sanitario, pueda plantear ante los CEA, la valoración del supuesto concreto sobre el estado de necesidad, con indicación incluso, si cabe, de la información que a su juicio se puede facilitar al paciente y cuál no. Con fundamento en dicha decisión, corresponde al médico tomar la decisión última dado que de acuerdo con la LAP es

\footnotetext{
${ }^{13}$ Las discusiones personales entre amigos médicos giran en torno al miedo que genera la falta de requisitos técnicos para aplicar este tipo de criterios.

${ }^{14}$ Los Comités de ética Asistencial fueron regulados por la Circular 3/1995, de creación y acreditación de comités asistenciales de ética.
}

REVISTA VIA INVENIENDI ET IUDICANDI, VOL. 8, No. 2

UNIVERSIDAD SANTO TOMÁS, BOGOTÁ

ISSN 1909-0528 
a él a quien corresponde determinar si se excluye al paciente o no de su derecho a conocer la información sobre su estado de salud y posibilidades terapéuticas de acuerdo a sospecha la objetiva de estar una situación de ENT. A la sazón, los CEA se convierten en una posibilidad consultiva o de apoyo a la decisión del profesional sanitario, más no pueden ser la fuente de la decisión de limitar el acceso a la información del enfermo ${ }^{15}$. En todo caso, cualquier valoración distinta que el médico realice en relación con el informe que la CEA emita, debe motivarla en razones objetivas que a su juicio justifiquen los cambios de criterio.

Para finalizar, el estudio de este punto es preciso decir que el estado de necesidad terapéutica, constituye un límite al deber de información del paciente, más no al derecho de acceso a la información contenida en la Historia Clínica. La primera no faculta el conocimiento de la información diversa, pero sí genera en quien tiene la custodia de la historia clínica un especial cuidado en el manejo de la información contenida en ella pues, la excepción por el estado de necesidad terapéutica debe quedar anotada en la Historia Clínica y esa parte, se deduce por simple lógica, sí debe estar restringida al paciente sin detrimento de los demás datos que obren en ella.

\section{Anotaciones subjetivas}

La excepción hecha a la entrega de información a los pacientes cuando hablamos de anotaciones subjetivas se refiere a un término legal nuevo que ha tratado las tantas veces citada LAP. Esta posibilidad no estaba contemplada en las legislaciones anteriores, especialmente la Ley 14/1986, de 25 de abril, General de Sanidad, no se limitaba el acceso del paciente a su información clínica (de acuerdo a la información del punto antes visto podría pensarse en el privilegio terapéutico como única excepción). Antes bien, el Real Decreto 63/1995 de 20 de enero, de Ordenación de Prestaciones Sanitarias del Sistema Nacional de Salud que

\footnotetext{
${ }^{15}$ Ver, por ejemplo para profundizar el tema: http://www.aceb.org/comit.htm
} 
desarrolla la Ley 14/1986, preveía la entrega íntegra, sin más, de la Historia Clínica al paciente cuando así lo solicitara.

El artículo 18.3 de la LAP, concreta una serie de limitaciones al derecho de acceso de información clínica del paciente. Una de factor objetivo (estado de necesidad terapéutica) que hemos tratado en el punto anterior. $Y$ otra de carácter subjetivo que es el correspondiente a este punto. El precepto citado determina que:

“...el derecho de acceso del paciente a la documentación de la historia clínica no puede ejercitarse en perjuicio del derecho de terceras personas a la confidencialidad de los datos que constan en ella recogidos en interés terapéutico del paciente, ni en perjuicio del derecho de los profesionales participantes en su elaboración, los cuales pueden oponer al derecho de acceso la reserva de sus anotaciones subjetivas.

De la lectura del precepto es posible destacar tres limitaciones claras, a saber: a) el interés de terceras personas, b) la necesidad terapéutica y c) las anotaciones subjetivas.

Por centrarnos en el estudio de este punto, debo destacar que la LAP, no ha definido qué se debe entender por el término de "anotaciones subjetivas". La información arrojada por la citada norma es más bien escasa, y la delimitación de su contenido ha dado lugar a un importante debate sobre su aplicabilidad, aunado al hecho de estar ante la limitación de un derecho fundamental: el derecho de información personal en una de sus dimensiones.

Como se trata de una excepción legal a un derecho fundamental, debe entenderse en su más restringido significado, o como lo recuerda el profesor GIL MEMBRADO, en su mínima expresión. Cualquier conflicto entre la interpretación del contenido y

REVISTA VIA INVENIENDI ET IUDICANDI, VOL. 8, No. 2 
significado de una anotación subjetiva, es deber que prevalezca el interés superior del paciente.

Ya habían dado luces sobre el particular el ilustre profesor Carlos María Romeo Casabona, cuando a principios de los años noventas al referirse a los componentes de la Historia clínica, mencionan aquellos datos que se incluyen en la misma y que derivan de una actividad subjetiva del médico. De acuerdo con el citado autor "habría que añadir como otro componente más las anotaciones subjetivas del médico con relación a las reacciones y actitudes del paciente, que son de especial importancia en algunos casos, como el tratamiento psiquiátrico. Sólo los mencionados en tercer lugar son susceptibles de ser considerados como creación científica objeto del derecho de propiedad intelectual, del que es titular el médico o médicos que ha participado directamente en su creación" (Casabona y Castellano, 1993: 14). De lo expuesto es preciso realizar el siguiente análisis.

El profesor Romeo Casabona apuntó en alguna ocasión anterior a una propuesta distinta. Par el citado profesor, el parámetro para saber si se trata de una anotación subjetiva depende, en ocasiones, de las reglas que regulan la propiedad intelectual pero no la considera del todo conveniente. De esta manera, recuerda que este derecho (el de propiedad intelectual) se puede ejercer directamente o por interpuesta persona, es decir podría ser direccionado por la política comercial de una empresa. Entonces, desde esta perspectiva es claro que se admitiría también que sean las entidades las que valoren las anotaciones subjetivas cuando en el contrato que lo vincula con el médico tratante, así estuviera incluido. Sin embargo es un debate que no corresponde ahora mismo emprender en el trabajo que se presenta.

Por su parte, en el sentido contrario, se ha manifestado la Agencia Española de Protección de Datos (en adelante, AEPD), quien en relación con las anotaciones subjetivas, ha indicado que una anotación subjetiva es: 
“.....toda aquella documentación o registro que en su origen no está destinado a informar al paciente y/o a un tercero relacionado con el proceso asistencial, o toda aquella elaboración personal que no esté soportada o acompañada de un registro de hechos medibles y por tanto constatable, estimando como "información objetiva" todo aquel contenido en los informes médicos, de enfermería y exploraciones complementarias realizadas". Apunte importante si se tiene en cuenta que quien realmente valora al paciente $\mathrm{y}$, por tanto, puede apreciar las circunstancias de cada caso es el médico ${ }^{16}$.

La doctrina de la Agencia Española de Protección de Datos, determina que la acreditación de que nos encontramos ante una anotación subjetiva le corresponde realizarla al médico tratante y nunca a la institución sanitaria. Desde mi punto de vista, esta posición resulta muy lógica, dado que evita una posible limitación al derecho de acceder a la información como en el caso que se analizó en la anterior resolución en la cual el motivo del hospital de Cabueñes era evitarse una posible demanda de responsabilidad. Por esta razón, considero a los pronunciamientos de la AEPD acertados, pues de lo contrario, si se permitiese a los Hospitales y Clínicas fijar los criterios de determinación de una anotación subjetiva, se permitiría que éstas hagan uso de una herramienta importante que les salvaría de demandas de responsabilidad médica en detrimento del derecho de los pacientes de conocer la evolución de su estado de salud o, en dado caso de acudir a un medio de defensa judicial necesario en este tipo de procesos.

En consecuencia, depende de cada profesional sanitario (no exclusivamente los médicos) más no de una entidad, determinar los casos en los cuales se encuentra

\footnotetext{
${ }^{16}$ RESOLUCIÓN R/00633/2004 AEPD, de 26 de mayo.
} 
ante una anotación subjetiva. La citada posición encuentra también apoyo en lo dispuesto por las distintas normas autonómicas sobre anotaciones subjetivas (enumeradas a continuación) ya que, en esencia, la LAP, al ser una norma básica, no define estrictamente a qué se refiere cuando habla de las anotaciones subjetivas y en este sentido deja un margen de obra para que sea cada caso concreto el que delimite esta situación. Por tratar algunas normas de las Comunidades Autónomas españolas que han regulado el tema, encontramos que el legislador autonómico en cada caso ha intentado delimitar su contenido, pero no han dado una definición precisa que determine su aplicabilidad. Sin embargo permiten orientar el contenido de lo que debe ser una AS.

El desarrollo que se ha dado por algunas de las Comunidades Autónomas (CCAA) está más o menos descrito de la siguiente manera:

La Ley 3 de 2005 de la CCAA de Extremadura en el art. 32.4.d. las define: se entenderán por anotaciones subjetivas las impresiones de los profesionales sanitarios, basadas en la exclusiva percepción de aquellos, y que, en todo caso, carecen de trascendencia para el conocimiento veraz y actualizado del estado de salud del paciente, sin que puedan tener consideración de un diagnóstico.

El Decreto 101 de 2005 de la CCAA de Castilla y León en el art. 6.b.: las anotaciones subjetivas de los profesionales que intervengan en el proceso asistencial deberán quedar claramente identificadas respecto del resto de la información contenida en la historia clínica, con el fin de facilitar su disociación cuando ésta sea precisa.

El Decreto 29 de 2009 de la CCAA de Galicia, en el art. 21.1. dice que son: las valoraciones personales, sustentadas o no en los datos clínicos de que se disponga en ese momento, que no formando parte de la historia clínica actual del/la paciente o usuario/a, puedan influir en el diagnóstico y futuro tratamiento médico una vez constatadas. 
La resolución de la dirección gerencia del servicio de salud de Castilla-La Mancha, dice:

Se consideran anotaciones subjetivas únicamente aquellas que puedan encuadrarse en uno de los siguientes apartados:

- Valoraciones sobre hipótesis diagnósticas no demostradas.

- Sospechas acerca de incumplimientos terapéuticos.

- Sospechas de tratamientos no declarados.

- Sospechas de hábitos no reconocidos.

- Sospechas de haber sido víctima de malos tratos.

- Comportamientos insólitos.

La Ley 5 de 2010 CCAA de Castilla-La Mancha, en el art. 29.6.: se entenderán por anotaciones subjetivas las impresiones de los profesionales sanitarios no sustentadas directamente en datos objetivos o pruebas complementarias y que, en todo caso, resulten de interés para la atención sanitaria del paciente.

Y la Ley 17 de 2010 CCAA de Navarra, en el art. 64.4. dice que son las impresiones o valoraciones personales de los profesionales sanitarios no sustentadas directamente en datos subjetivos o pruebas complementarias y que, en su criterio, resulten de interés para la atención sanitaria del paciente.

Así, encontramos entonces algunos asuntos sobre los que giran las principales críticas al concepto de anotación subjetiva.

El recelo sobre las anotaciones subjetivas recurrente en los artículos y libros que sobre él han hablado está basado en la indeterminación del concepto y el peligro para el derecho de información del paciente que supone un mal uso de la restricción hecha a contenidos de la Historia clínica. 
Se discute si tal concepto ha de referirse en exclusiva a sospechas terapéuticas 0 sospechas relacionadas directa o indirectamente con el tratamiento o la asistencia clínica (sospechas de consumo de sustancias psicoativas, sospechas de sufrimiento de violencia intrafamiliar, etc) que el médico tiene y no ha sido revelada por el paciente, o criterios directamente relacionados con la enfermedad presentada por el paciente pero no diagnosticada con certeza por los datos relevados por éste, pues no todas las personas desarrollan el mismo nivel de confianza con su médico tratante.

Vemos que las regulaciones autonómicas han sido determinantes para entender qué debe considerarse como una anotación subjetiva y no correr el riesgo de limitar injustificadamente un derecho de carácter fundamental contenido en la CE.

Esta línea de argumentación legal ha sido importante por cuanto de los casos presentados a la AEPD se evidencia que en la mayoría de ellos se restringía injustificadamente el acceso a la información del paciente alegando, el mismo centro de salud, encontrarse ante anotaciones subjetivas. Por ello considero, en el mismo sentido que se ha manifestado la doctrina, no pueden considerarse anotaciones subjetivas a aquellas directamente relacionadas con los diagnósticos probados y la atención prestada sobre el estado de salud del paciente, pues el carácter subjetivo no se encuentra ligado a ninguna de ellas. Por más que sean valoraciones hechas por el equipo médico, su sustento está en la ciencia médica, actualmente llamada medicina basada en la evidencia.

Vemos por ejemplo lo que manifiesta el autor GIL MEMBRANO:

"se trataría, por lo tanto, según esta vertiente doctrinal, de impresiones subjetivas del profesional sobre el enfermo o sobre su entorno con trascendencia clínica y, que por ello, deben integrarse a la $\mathrm{HC}$ del paciente, pero que a diferencia de 
los resultados de las exploraciones, el juicio diagnóstico, el pronóstico, el tratamiento, etc... no constituyen parte del juicio clínico sino que son juicios de valores respecto a los que el paciente tiene restringido el acceso" (GIL MEMBRANO, 2010: 255).

\section{Conclusión}

Nos encontramos ante un supuesto en el que el límite del derecho y deber de información, en su forma estructural, difiere del anterior (Estado de Necesidad Terapéutica), pues este último tiene su contenido en valoraciones objetivas que finalizan en una situación de alarma generadora de un posible menor beneficio terapéutico al paciente, mientras que las AS son valoraciones subjetivas intrínsecas de cada médico o equipo médico que no tengan relación directa con la enfermedad o el tratamiento.

\section{Referencias}

FOMBELLA Posada, María José y CEREIJO Quinteiro, María José (2012); Historia de la Historia Clínica, en Galicia Clínica 2012; 73 (1): 21-26.

GALÁN CORTÉS, Julio César (2011). Responsabilidad civil médica. Tercera Edición. Editorial Civitas. Navarra - España.

GIL MEMBRADO, Cristina (2010); La Historia Clínica, Deberes del responsable del tratamiento y derechos del paciente; Editorial Comares. Granada, España.

OLIVA Blásquez, Francisco. Autonomía del paciente y sistema sanitario: conflicto de bienes jurídicos. Vía web: 
CORREA M., CÉSAR ALBERTO (2013): “LÍMITES AL DERECHO DE ACCESO A LA INFORMACIÓN CLÍNICA EN LOS CASOS DE ESTADO NECESIDAD TERAPÉUTICA Y ANOTACIONES SUBJETIAS: ESPECIAL REFERENCIA AL SISTEMA ESPAÑOL", VIA INVENIENDI ET IUDICANDI, VOL. 8, No. 2, PP. 33-52

http://www.juntadeandalucia.es/export/drupal cpre/XV Jornadas Ponencia Fran cisco Oliva.pdf. Recuperado: diciembre 5 de 2013.

RIPOLL CARULLA Santiago, BACARIA MARTRUS Jordi \& Otros. Estudios de Protección de Datos de carácter personal en el ámbito de la salud. Agencia Catalana de protección de datos APDCAT. Marcial Pons, ediciones jurídicas y sociales S.S., Madrid, 2006.

ROMEO Casabona, Carlos María y CASTELLANO Arroyo, María (1993); La intimidad del paciente desde la perspectiva del secreto médico y el acceso a la historia clínica. 